\title{
Factors lead to return to sports and recreational activity after total knee replacement
}

\section{A retrospective study}

Jeremy Plassard ${ }^{1,4, *}$, Jean Baptiste Masson ${ }^{1}$, Matthieu Malatray ${ }^{1}$, John Swan ${ }^{1}$, Francesco Luceri ${ }^{5,6}$, Julien Roger ${ }^{1}$, Cécile Batailler ${ }^{1}$, Elvire Servien ${ }^{1,3}$, and Sébastien Lustig ${ }^{1,2}$

${ }^{1}$ FIFA Medical Center of Excellence, Department of Orthopaedic Surgery and Sports Medicine, Croix Rousse Hospital, Civil Hospices of Lyon, 69004 Lyon, France

2 LBMC UMR T 9406, Laboratory of Chock Mechanics and Biomechanics, Claude Bernard Lyon 1 University, 69100 Villeurbanne, France

3 LIBM - EA 7424, Interuniversity Laboratory of Biology of Mobility, Claude Bernard Lyon 1 University, 69100 Villeurbanne, France

${ }^{4}$ Department of Orthopedic and Traumatology, Dijon University Hospital, 21000 Dijon, France

5 Clinica Ortopedica, ASST Centro Specialistico Ortopedico Traumatologico Gaetano Pini-CTO, Piazza Cardinal Ferrari 1, 20122 Milan, Italy

${ }^{6}$ Laboratory of Applied Biomechanics, Department of Biomedical Sciences for Health, Università degli Studi di Milano, Via Mangiagalli 31, 20133 Milan, Italy

Received 13 April 2020, Accepted 16 April 2020, Published online 7 May 2020

\begin{abstract}
Introduction: The number of total knee replacements performed (TKR) is increasing and so are patient expectations and functional demands. The mean age at which orthopedic surgeons may indicate TKR is decreasing, and therefore return to sport (RTS) after TKR is often an important expectation for patients. The aim of this study was to analyze the mid-term RTS, recreational activities, satisfaction level, and forgotten joint level after TKR. Methods: Between January 2015 and December 2016, 536 TKR (same implant design, same technique) were performed in our center. The mean age at survey was 69 years with a mean follow-up of 43 months. All patients who did not have a follow-up in the last 6 months were called. Finally, 443 TKR were analyzed. RTS was assessed using the University of California Los Angeles Scale (UCLA), forgotten joint score (FJS), and Satisfaction Score. Results: In this study, $85 \%$ of patients had RTS after TKR with a mean UCLA score increasing from 4.48 to 5.92 and a high satisfaction rate. Satisfaction with activity level was $93 \%$ (satisfied and very satisfied patients). The RTS is more important for people with a higher preoperative UCLA score and a lower American Society of Anesthesiologist score (ASA). Each point increase in ASA score is associated with reduced probability to RTS by $52 \%$. Discussion: RTS and recreational activity were likely after TKR with a high satisfaction score. Preoperative condition and activity are the two most significant predictive factors for RTS. Level of evidence: Retrospective case series, level IV.
\end{abstract}

Key words: Total knee replacement, Return to sport, Recreational activities, Patient satisfaction.

\section{Introduction}

According to demographic projections, the number of patients suffering from osteoarthritis (OA) will increase dramatically in the future, and therefore the number of total knee replacement (TKR) too. This increase is multifactorial: improved life expectancy, increasing obesity, and younger patients with knee OA secondary to trauma or other reasons. The younger population often expects to maintain an active lifestyle without pain, or stiffness.

OA restricted the ability to carry out daily activities, work, and perform sport activities. Pain and decreased range of motion

\footnotetext{
*Corresponding author: jeremyplassard@live.fr
}

lead to physical deconditioning with reduced endurance, less aerobic capacity, and a higher risk of being overweight and developing cardiovascular diseases. Ravi et al. [1] reported how the management of knee OA with arthroplasty may have a role in cardiovascular prevention. Furthermore, TKR is a cost-effective surgical procedure, especially in young and active patients [2].

TKR provides functional improvement in patients with advanced knee OA [3, 4]. Although evidence suggests a full return to active life after TKR is possible [5, 6], many orthopedic surgeons still prefer to advise limited sporting activities postoperatively.

In fact, pain reduction is often insufficient with young highly demanding patients with OA wanting to return to the same 


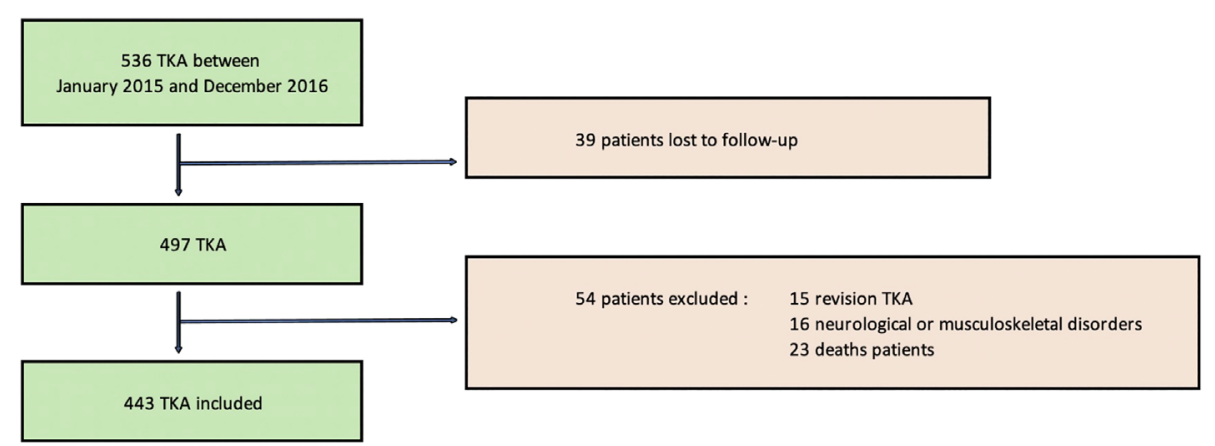

Figure 1. Flowchart of the study (RTKR: revision TKR).

pre-arthritic level of sport. These patients do not only aim for pain relief after TKR. With innovative surgical techniques and more anatomical implants, TKR is now indicated in younger patients with higher sports expectations after TKR [7]. Nowadays it is becoming more important to be able to predict the level of postoperative sport activity for each specific patient. Bullens et al. [4] and Nobel et al. [8] compared subjective and objective outcomes after TKR, and notably, satisfaction depends on preoperative expectations, which are correlated with objective results.

The aim of this study was to analyze the mid-term return to sport (RTS) and recreational activities, satisfaction level, and forgotten joint level, and to find preoperative factors associated to better functional outcomes after TKR, then, to know whether these factors could allow a better preoperative information to patients about RTS and expected activities after TKR.

\section{Material and methods}

\section{Study design}

There were 536 patients enrolled in this retrospective study who underwent TKR between January 2015 and December 2016. Surgery was performed using the same primary highflexion posterior-stabilized cemented implant (Anatomic $^{\circledR}$, Amplitude, France).

TKR was performed without tourniquet using the same surgical technique by four different senior surgeons. A medial parapatellar trans-quadricipital approach was performed for varus knees and the Keblish lateral approach was performed for valgus knees. The patella was resurfaced only when severe patellar osteoarthritis was present. An immediate full-weightbearing rehabilitation protocol was used with thromboembolic prophylaxis postoperatively for 30 days in all patients.

The inclusion criteria were primary TKR for symptomatic knee osteoarthritis. A total of 536 TKR were performed between January 2015 and December 2016. The exclusion criteria were: revision TKR, inflammatory osteoarthritis, neuromuscular conditions (Parkinson's disease, Alzheimer's disease, stroke), or conditions that may interfere with the standard postoperative rehabilitation protocol. We excluded 15 revision TKR, 16 patients with neurological or musculoskeletal disorders and 23 deaths at the time of final follow-up. All patients without clinical follow-up in the last 6 months were evaluated using a functional questionnaire. Finally 39 patients (7\%) were lost to
Table 1. Sample characteristics and outcomes.

\begin{tabular}{lc}
\hline Age (years) & $69(41-90)$ \\
Age $<65$ yo & $146(41-65)$ \\
Age $>65$ yo & $297(66-90)$ \\
Gender & \\
Male & $162(36.6 \%)$ \\
Female & $281(63.4 \%)$ \\
BMI & $29.3(19-46)$ \\
Knee surgery before & \\
Medial meniscectomy & $58(13 \%)$ \\
Articular washing & $38(8.5 \%)$ \\
HTO & $32(7 \%)$ \\
ACL ligamentoplasty & $27(6 \%)$ \\
Lateral meniscectomy & $24(5.5 \%)$ \\
Tibial plate fracture & $10(2.2 \%)$ \\
Temporal fracture & $4(1 \%)$ \\
DFO & $1(0.2 \%)$ \\
Varus deformity & $311(70 \%)$ \\
Valgus deformity & $87(20 \%)$ \\
Normoaxis & $45(10 \%)$ \\
IKS objective & 47 \\
UCLA < 4 & $119(27 \%)$ \\
UCLA 4-6 & $289(65 \%)$ \\
UCLA $>6$ & $35(8 \%)$ \\
\hline
\end{tabular}

follow-up (non-responders or contact details where changed). Four hundred and forty three TKR were included in the study. Patient flow-chart is presented in Figure 1 and demographic characteristics are summarized in Table 1. The mean followup in this study was 43 months [23-49 months].

Patients completed the questionnaire at 2 months, 1-year follow-up, and every 2 years postoperatively. Before surgery activity level was assessed using the UCLA score [3,9] and sport type by direct question. All patients who did not have face-to-face consultation in 2019 were called. We analyzed the postoperative UCLA score: activity level: low $(\leq 3)$, moderate (4-6), and high $(\geq 7)$ [10]. RTS after surgery (in months), type of sport most frequently performed after surgery, forgotten joint score (FJS) [11] and a satisfaction score (very satisfied, satisfied, disappointed, dissatisfied) based on the new IKS score [12] were also collected in the study.

\section{Statistical analysis}

Patient demographics were described using means and standard deviations or medians and ranges for continuous variables 
and percentage counts for categorical variables. The study sample was divided into four sub-groups according to sex and age: younger group (age $\leq 65$ yo) and older group (age $>65$ yo). Preoperative and postoperative sport level, RTS, FJS, and the level of satisfaction were compared between groups. A multivariate linear regression analysis was performed to assess possible relationships between return to sport and the included variables: age at time of surgery, ASA score, sex, body mass index (BMI), preoperative UCLA group (low, moderate, or high), type of deformity (neutral, varus, or valgus alignment groups), degree of deformity, and patellar resurfacing. We used a generalized binomial linear logistic model produced under $\mathrm{R}$ Commander for multivariate analysis.

\section{Results}

\section{Return to sport}

A total of 376 patients $(85 \%)$ returned to sport after TKR, 240 women (85\%) and 136 men (84\%). This represents $88 \%$ patients in the $\leq 65$ yo group (129 patients) and $83 \%$ in the $>65$ yo group (247 patients). The mean delay to RTS was 5 months [1-36 months], with $33 \%$ of the patients returning to sport or activities within 3 months postoperatively and $81 \%$ of the patients within 6 months postoperatively.

\section{Predictive factors to RTS}

Multivariate analysis shows a significant difference in return to sport according to preoperative UCLA scores $(p<0.001)$. Patients with a higher preoperative UCLA score had a higher RTS rate. A higher ASA score was a negative predictive factor for RTS $(p<0.005)$, with each increase in ASA of one point being associated with a reduction of RTS probability by $52 \%$. There were no significant differences in age, sex, BMI $(p=0.054)$, severity of preoperative knee deformity, previous knee surgery history, and patellar resurfacing.

\section{Level of activity: UCLA}

All patients were divided into three groups for activity level: low activity group (UCLA score $\leq 3$ ), moderate group (UCLA score 4-6), and high active group (UCLA scale $\geq 7$ ) (Figure 2); and in two groups for sex and age: $\leq 65$ years and $>65$ years (Figure 3). The mean preoperative UCLA score was 4.45 and the mean postoperative UCLA score was 5.92, the mean UCLA activity score increased by 1.47 point. Compared to preoperative UCLA scores, 357 patients (80\%) had a significant postoperative improvement, 64 patients $(14 \%)$ achieved the same score, and 22 patients $(5 \%)$ had a decreased UCLA score (1-3 UCLA points). After TKR, $82 \%$ of patients declared being RTS or recreational activities, with only 67 patients declaring restriction in their daily activities due to their TKR.

\section{Satisfaction and forgotten joint score (FJS)}

At the last follow-up, the satisfaction rate after TKR was important. Four hundred and twelve patients reported that they

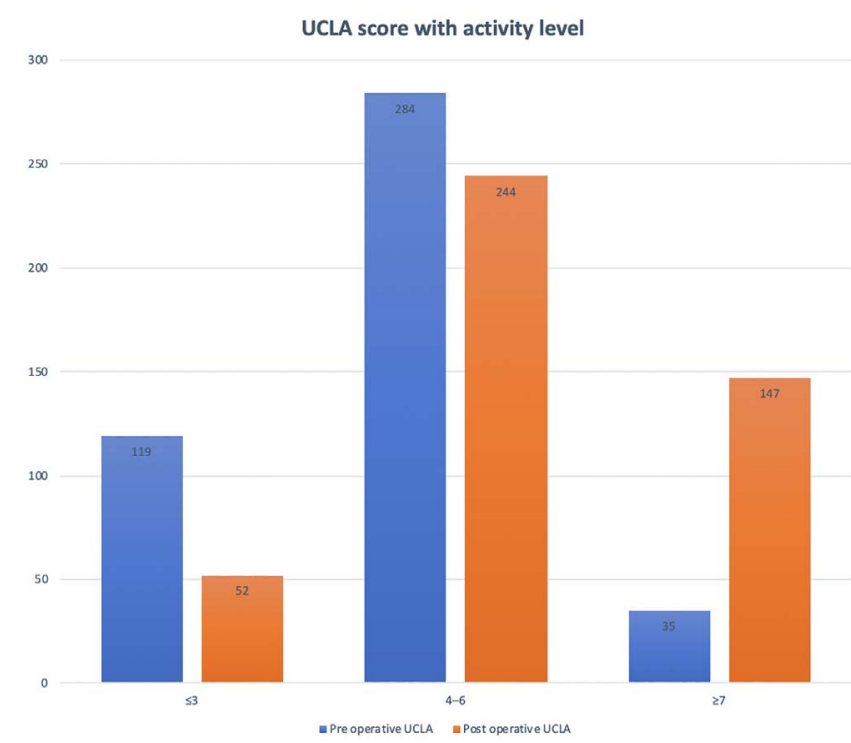

Figure 2. Pre- and post-operative UCLA score with activity level.

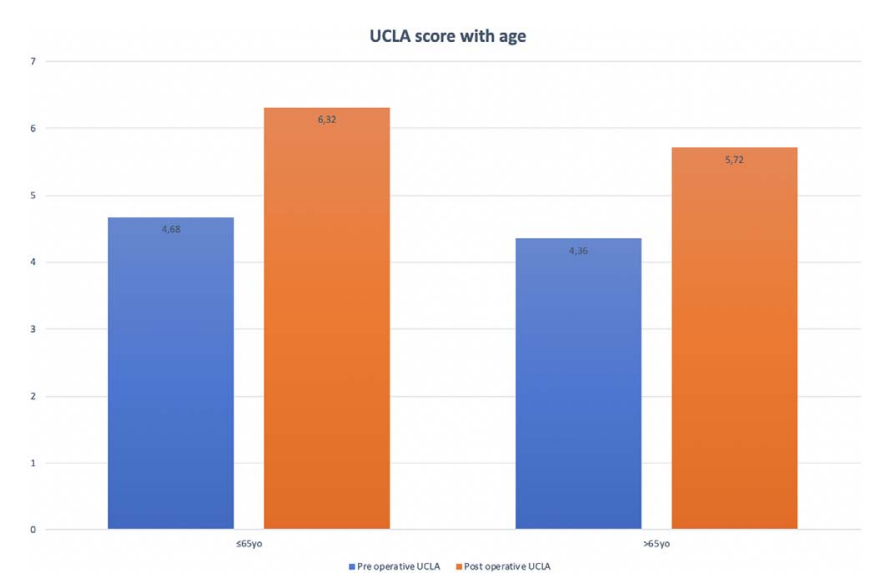

Figure 3. Pre- and post-operative UCLA score with age.

were satisfied or very satisfied (93\%), 17 were moderately satisfied (4\%), and 15 were unsatisfied (3\%). Unsatisfied patients had a lower FJS level, down to 25/100 and only 12 patients returned to sport. 344 patients $(77 \%)$ had a FJS higher than $75 / 100$ and no limitations in physical activity and their satisfaction rate was up to $38 / 40$. There were no significative differences in postoperative satisfaction between gender and age.

\section{Sport disciplines}

The most frequently performed activities were walking, hiking, gardening, swimming, yoga, cycling, and playing golf (Table 2). Three hundred and seventy six patients were involved in sports activities, 326 patients (86.9\%) reported achieving a higher level. 39 patients reported being at the same level $(10.3 \%)$ and 11 patients $(3 \%)$ at a lower level compared to preoperative levels. Low-impact activities such as walking, hiking, cycling, or swimming were performed more after surgery and we found a decrease in high-impact activities such 
Table 2. Variation in types of sport practiced.

\begin{tabular}{lcc}
\hline & Pre operative & Post operative \\
\hline Walking & $208(46.9 \%)$ & $263(59.3 \%)$ \\
Cycling & $106(23.9 \%)$ & $120(27.0 \%)$ \\
Swimming & $47(10.6 \%)$ & $56(12.6 \%)$ \\
Gardening & $50(11.3 \%)$ & $55(12.4 \%)$ \\
Hiking & $48(10.8 \%)$ & $54(12.2 \%)$ \\
Fitness/yoga & $26(5.8 \%)$ & $32(7.2 \%)$ \\
Skiing & $28(6.3 \%)$ & $23(5.2 \%)$ \\
Golf & $11(2.4 \%)$ & $9(2.0 \%)$ \\
Petanque & $6(1.3 \%)$ & $6(1.3 \%)$ \\
Fishing/hunting & $4(0.9 \%)$ & $5(1.2 \%)$ \\
Tennis & $5(1.2 \%)$ & $3(0.7 \%)$ \\
Dancing & 0 & $2(0.5 \%)$ \\
Running & $4(0.9 \%)$ & $1(0.3 \%)$ \\
Climbing & $1(0.3 \%)$ & $1(0.3 \%)$ \\
\hline
\end{tabular}

as tennis, jogging, or skiing. Patients with a lower level of sporting activity cited reasons such as precautionary avoidance to preserve their TKR (nine patients), knee pain (seven patients), general health condition, two patients with the sensation of knee instability, and two patients with periprosthetic infection.

\section{Range of motion evolution}

We analyzed for an association between range of motion (ROM) and patient satisfaction. In unsatisfied, low-satisfied, and satisfied groups, the range of motion decreased after TKR: from $117^{\circ}$ to $109^{\circ}$ for unsatisfied patients, from $122^{\circ}$ to $114^{\circ}$ for low-satisfied patients, and from $125^{\circ}$ to $122^{\circ}$ for satisfied patients. Only highly satisfied patients had an increase in ROM after TKR from $120^{\circ}$ to $122^{\circ}$.

\section{Complications}

There were eight TKR revision surgeries: two for aseptic loosening, three for prosthetic joint infection (PJI), two patellar revisions for lateral patellar instability, and one for patellar clunk syndrome. Five of the eight patients who required revision surgery managed to return to sports prior to revision.

\section{Discussion}

The principle finding of this study was that most patients returned to sport by 6 months postoperatively (80\%), with a significantly higher UCLA score than prior to surgery. Therefore, patients should expect participation in sports activities after TKR.

The most reliable predictive factors of RTS after TKR are preoperative UCLA and ASA scores and, contrary to other published studies $(15,16,20)$, age, gender, and BMI appear not to influence the RTS. The absolute number of sports practiced by patients pre- and postoperatively was similar; however, there was a change in types of physical activities. This study was not the first to evaluate the RTS after TKR, but it is one of the largest and with long-term follow-up. Ten studies were used to compare to our series (Table 3). These studies had a mean follow-up >1 year, more than 100 patients (except [13]), and meta-analysis. Bradbury et al. [14] reported that $77 \%$ of patients who participated in sport preoperatively returned to sport postoperatively with or without adaptation of activities. More recently, Chatterji et al. [15] reported that $75 \%$ of patients returned to sports activities after 1 year.

Mean postoperative UCLA score was comparable to the study by Bauman et al. [16] who found a mean score of 6.0 in a series of 184 TKR. Dahm et al. [3] reported a mean score of 7.1 with $74 \%$ of patients engaged in activities at a mean of 5.7 years after arthroplasty, with $16 \%$ of the patients reporting participating in heavy manual labor or sports deemed "not recommended" by the Knee Society survey [6].

Chatterji et al. [15] and Wylde et al. [17] have found, patients who underwent TKR reduced the intensity of highimpact activities such as jogging, skiing, or tennis, while increasing low-impact activities such as walking, hiking, cycling, or swimming. In a systematic review, Witjes et al. [18] reported that RTS for TKR varied from $36 \%$ to $89 \%$, with mean total numbers of postoperative sports of $0.2-1.0$ sports with a mean of 13 weeks for RTS.

There is no evidence of any correlation between high-level sports and early implant loosening, bearing surface wear or premature revision. Although some surgeons discourage highimpact sport, in contrast, Mont et al. [19] showed that highlevel tennis players returned to tennis after TKR. Healy et al. [6] reported limited evidence-based information on implant survival after sport to make recommendations where patients are recommended to avoid high-impact sports. In our study 147 patients $(33 \%)$ participated in high-impact sport without implant failure. Therefore, if patients understand the risks associated with their activities and choose to return to sport, surgeon should not discourage their patients. Kersten et al. [20] showed that almost half of patients who underwent TKR did not meet health-enhancing physical activities guidelines. In a study by Walker et al. [21] of patients with lateral unicompartmental knee replacements (UKR), the majority of patients decreased their activities to protect their UKR. In our series of patients with TKR, activity restriction because of the TKR occurred in only 22 patients for reasons of pain, knee instability, or limited range of motion.

Hopper and Leach [13] compared UKR to TKR, where UKR had superior results and they found that patients with TKR returned to low-impact sport. In the TKR group, $63.4 \%$ returned to sport by 4.1 months with a significant reduction in playing bowls and golf postoperatively. In active golfers, Mallon and Callaghan [22] found that the players' handicap increased significantly and their driving distance was substantially reduced. Jones et al. [23] did not show an increase in revision rates due to high-impact activities at midterm follow-up. Bonnin et al. [24] have shown how satisfaction depends upon preoperative patient expectations. In our study, we have a high percentage of patients that are satisfied or very satisfied (92\%) with a mean Forgotten Joint Score of $82 / 100$. This demonstrates the improvement in quality of life as a result of TKR, which is confirmed by other studies [25]. 
Table 3. Return to sport after TKR, data extracted from studies included in the review (10 studies).

\begin{tabular}{|c|c|c|c|c|c|c|c|c|}
\hline Study & Study design & $\begin{array}{c}\text { Mean } \\
\text { follow-up }\end{array}$ & $\begin{array}{c}\text { Study } \\
\text { population }\end{array}$ & RTS & $\begin{array}{c}\text { Time to } \\
\text { RTS }\end{array}$ & $\begin{array}{l}\text { Mean post- } \\
\text { operative } \\
\text { UCLA } \\
\end{array}$ & $\begin{array}{c}\text { UCLA } \\
\text { progression }\end{array}$ & $\begin{array}{l}\text { Satisfied } \\
\text { and very } \\
\text { satisfied }\end{array}$ \\
\hline Bauman et al. [16] & Cross-sectional survey & 36.6 month & 184 patients & - & - & 6 & - & - \\
\hline Bonnin et al. [24] & Cross-sectional survey & 44 month & 347 patients & $\begin{array}{c}56 \% \text { group } \\
<75 \text { yo }\end{array}$ & - & - & - & $83 \%$ \\
\hline Bradbury et al. [14] & Cross-sectional survey & 5 years & 160 patients & $77 \%$ & - & - & - & - \\
\hline Chang et al. [10] & Retrospective study & 2 years & 369 patients & $76 \%$ & - & 4.8 & +0.3 & - \\
\hline Chatterji et al. [15] & Cross-sectional survey & $\begin{array}{c}\text { Between } \\
1 \text { and } 2 \text { years }\end{array}$ & 144 patients & $75 \%$ & - & - & - & - \\
\hline Dahm et al. [3] & Cross-sectional survey & 5.7 years & 1206 patients & - & - & 7.1 & - & $91 \%$ \\
\hline Hopper and Leach [13] & Cross-sectional survey & 21.6 months & 76 patients & $63 \%$ & - & - & - & $80.3 \%$ \\
\hline Noble et al. [8] & Cross-sectional survey & $>1$ year & 253 patients & - & - & - & - & $75 \%$ \\
\hline Witjes et al. [18] & Systematic review & Variable & 18 studies & $36-89 \%$ & 12 weeks & $4.6-5.9$ & - & - \\
\hline Wylde et al. [17] & Cross-sectional survey & $>3$ years & 866 patients & $73 \%$ & - & - & - & - \\
\hline
\end{tabular}

This study has several limitations. Firstly, this work is a retrospective study, with a low level of evidence. Secondly, $7 \%$ of patients were lost to follow-up, and we could not assess if these patients were still alive or if they underwent revision surgery. In a similar study, Chang et al. [10] had a response rate of just $65 \%$. Thirdly, the mean follow-up in this study was 43 months, which is adequate to determine early satisfaction outcomes, activity levels and time to RTS, but not long enough to evaluate revision rates, wear, and loosening.

Total knee arthroplasty is an effective treatment for pain relief; functional improvement and RTS is likely after TKR with a high satisfaction score. Preoperative condition and activity are the two most significant predictive factors for RTS. After TKR, patients should be encouraged to maintain physical activities with individual adaptions based upon general health, preoperative activity level, and type of preoperative sport. Then, surgeons should explain to the patient what kind of activities they can participate in after TKR and how to adjust their activities and give more details on expected results according to patients' preoperative status and health.

\section{Conflicts of interest}

JP, JBM, MM, JS, FL, JR, CB, and ES declare that they have no conflict of interest with this study. SL declares the following conflicts of interest: consulting (Smith and Nephew, Stryker, Groupe Lepine, Aeraeus, Medacta, Amplitude, Corin), institutional research support to Corin and Amplitude.

\section{References}

1. Ravi B, Croxford R, Austin PC, et al. (2014) The relation between total joint arthroplasty and risk for serious cardiovascular events in patients with moderate-severe osteoarthritis: Propensity score matched landmark analysis. Br J Sports Med 48, 1580.

2. Bedair H, Cha TD, Hansen VJ (2014) Economic benefit to society at large of total knee arthroplasty in younger patients: A Markov analysis. J Bone Joint Surg Am 96, 119-126.
3. Dahm DL, Barnes SA, Harrington JR, et al. (2008) Patientreported activity level after total knee arthroplasty. J Arthroplasty 23, 401-407.

4. Bullens PH, van Loon CJ, de Waal Malefijt MC, et al. (2001) Patient satisfaction after total knee arthroplasty: A comparison between subjective and objective outcome assessments. J Arthroplasty 16, 740-747.

5. Swanson EA, Schmalzried TP, Dorey FJ (2009) Activity recommendations after total hip and knee arthroplasty: A survey of the American Association for Hip and Knee Surgeons. J Arthroplasty 24, 120-126.

6. Healy WL, Iorio R, Lemos MJ (2000) Athletic activity after total knee arthroplasty. Clin Orthop Relat Res 90, 65-71.

7. Crowninshield RD, Rosenberg AG, Sporer SM (2006) Changing demographics of patients with total joint replacement. Clin Orthop Relat Res 443, 266-272.

8. Noble PC, Conditt MA, Cook KF, Mathis KB (2006) The John Insall Award: Patient expectations affect satisfaction with total knee arthroplasty. Clin Orthop Relat Res 452, 35-43.

9. Naal FD, Impellizzeri FM, Leunig M (2009) Which is the best activity rating scale for patients undergoing total joint arthroplasty? Clin Orthop Relat Res 467, 958-965.

10. Chang MJ, Kim SH, Kang YG, et al. (2014) Activity levels and participation in physical activities by Korean patients following total knee arthroplasty. BMC Musculoskelet Disord 15, 240.

11. Behrend H, Giesinger K, Giesinger JM, Kuster MS (2012) The "Forgotten Joint" as the ultimate goal in joint arthroplasty. J Arthroplasty 27, 430-436.e1.

12. Scuderi GR, Bourne RB, Noble PC, et al. (2012) The new knee society knee scoring system. Clin Orthop Relat Res ${ }^{\circledR} 470,3-19$.

13. Hopper GP, Leach WJ (2008) Participation in sporting activities following knee replacement: Total versus unicompartmental. Knee Surg Sports Traumatol Arthrosc 16, 973-979.

14. Bradbury N, Borton D, Spoo G, Cross MJ (1998) Participation in sports after total knee replacement. Am J Sports Med 26, 530-535.

15. Chatterji U, Ashworth MJ, Lewis PL, Dobson PJ (2005) Effect of total knee arthroplasty on recreational and sporting activity. ANZ J Surg 75, 405-408.

16. Bauman S, Williams D, Petruccelli D, et al. (2007) Physical activity after total joint replacement: A cross-sectional survey. Clin J Sport Med 17, 104-108. 
17. Wylde V, Blom A, Dieppe P, et al. (2008) Return to sport after joint replacement. J Bone Joint Surg Br 90, 920-923.

18. Witjes S, Gouttebarge V, Kuijer PPFM, et al. (2016) Return to sports and physical activity after total and unicondylar knee arthroplasty: A systematic review and meta-analysis. Sports Med 46, 269-292.

19. Mont MA, Marker DR, Seyler TM, et al. (2008) High-impact sports after total knee arthroplasty. J Arthroplasty 23, 80-84.

20. Kersten RFMR, Stevens M, van Raay JJAM, et al. (2012) Habitual physical activity after total knee replacement. Phys Ther 92, 1109-1116.

21. Walker T, Gotterbarm T, Bruckner T, et al. (2015) Return to sports, recreational activity and patient-reported outcomes after lateral unicompartmental knee arthroplasty. Knee Surg Sports Traumatol Arthrosc 23, 3281-3287.
22. Mallon WJ, Callaghan JJ (1993) Total knee arthroplasty in active golfers. J Arthroplasty 8, 299-306.

23. Jones DL, Cauley JA, Kriska AM, et al. (2004) Physical activity and risk of revision total knee arthroplasty in individuals with knee osteoarthritis: A matched case-control study. J Rheumatol 31, 1384-1390.

24. Bonnin M, Laurent JR, Parratte S, et al. (2010) Can patients really do sport after TKA? Knee Surg Sports Traumatol Arthrosc 18, 853-862.

25. Ethgen O, Bruyère O, Richy F, et al. (2004) Health-related quality of life in total hip and total knee arthroplasty. A qualitative and systematic review of the literature. J Bone Joint Surg Am 86, 963-974.

Cite this article as: Plassard J, Masson J, Malatray M, Swan J, Luceri F, Roger J, Batailler C, Servien E \& Lustig S (2020) Factors lead to return to sports and recreational activity after total knee replacement. SICOT-J 6, 11 